\title{
Dental Avulsion- Immediate Replantation: 8- Year Follow UP Case
}

\author{
Hanali Abu Shilbayih*,Nezar Watted**, Azzaldeen Abdulgani***, Muhamad \\ Abu-Hussein $* * * *$ \\ * Department Of Orthodontics And Pediatric Dentistry, Al-Quds University, Jerusalem, Palestine \\ **University Hospital Of Würzburg Clinics And Policlinics For Dental, Oral And Maxillofacial Diseases Of \\ The Bavarian Julius-Maximilian- University Wuerzburg, Germany And Center For Dentistry Research And \\ Aesthetics, Jatt, Israel \\ *** Department Of Conservative Dentistry, Al-Quds University, Jerusalem, Palestine \\ ****Department Of Pediatric Dentistry, University Of Athens, Greece
}

\begin{abstract}
Avulsion of permanent front teeth is a rare accident, mostly affecting children between seven and nine years of age. Replanted and splinted, these teeth often develop inflammation, severe resorption or ankylosis affecting alveolar bone development and have to be extracted sooner or later. This report proposes a discussion on the various peculiarities of a tooth avulsion case with immediate

replantation, such as a long retention period, root canal filling with MTA, orthodontic treatment .
\end{abstract}

Keywords; Avulsion, mineral trioxide aggregate, tooth avulsion, tooth replantation

\section{Introduction}

Dental avulsion is the most severe type of traumatic tooth injuries because it causes damage to several structures and results in the complete displacement of the tooth from its socket in the alveolar bone. [1]The ideal situation is to replant an exarticulated tooth immediately after avulsion because the extraoral time is a determinant factor for treatment success and for a good prognosis. However, it is not always possible.[1,2]

The success of replantation depends on a number of factors that may contribute to

accelerate or minimize the occurrence of root resorption or ankylosis, among which is the type and characteristics of the medium used for temporary storage during the time elapsed between avulsion and replantation.[1-5]

The first reported cases of knocked-out teeth being replanted was by Pare' in 1593. In 1706, Pierre Fauchard also reported replanting knocked out teeth. Wigoper in 1933 used a cast gold splint to hold reimplanted teeth in place. In 1959, Lenstrup and Skieller declared that the success rate of replanted knocked out teeth should be considered a temporary procedure because the success rate of less than $10 \%$ was so poor.[6] In 1966 in a retrospective study, Andresen theorized that $90 \%$ of avulsed teeth could be successfully retained if they were replanted within the first 30 minutes of the accident.[7,8] In 1974, Cvek showed that removal of the dental pulp following reimplantation was necessary to prevent resorption of the tooth root. In 1974, Cvek showed that storage of knocked out teeth in saline could improve the success of replanted teeth.[9,10] In 1977, Lindskog et al. showed that the key to retention of the knocked-out teeth was to maintain the vitality of the periodontal ligament. In 1980, Blomlof showed the storing the periodontal ligament cells in a biocompatible medium could extend the extra oral time to four hours or more. He found that the best storage medium was a medical research fluid called Hank's Balanced Solution. In this study, it was serendipitously discovered that milk could also maintain cell viability for two hours.[11,12] In 1981, Andreasen showed that crushing of cells on the tooth root could cause death of the cells and lead to resorption and reduction in prognosis.[13] In 1983, Matsson et al. showed that soaking in Hank's Balanced Solution for thirty minutes prior to reimplantation could revitalize extracted dog's teeth that were dry for 60 minutes.[14,15] In 1989, a systematic storage device was developed to optimally store and preserve knocked out teeth. In 1992, Trope et al. showed that extracted dog's teeth could be stored in Hank's Balanced Solution for up to 96 hours and still maintain significant vitality. In this study, milk was only able to maintain vitality for two hours.[16]

Tooth avulsion is characterized as total displacement of the tooth out of its alveolar socket with damage to the periodontal ligament, cementum, alveolar bone, as well as gingival and pulpal tissues [2,3]. Its prevalence in permanent dentition is about $1 \%$ to $16 \%$ of traumatic injuries $[4,5]$. Avulsion of the permanent teeth occurs more frequently in children between 7 to 9 years in which the periodontal ligament structure is still loose and the surrounding bone of the newly erupted teeth is slightly mineralized. Thus, there is only a minimal resistance to the extrusive forces. Maxillary central incisors are more prone to avulsion $[4,5]$. 
The common cause of injury are falls, collisions and accidents during common childhood activities such as contact sports, cycling, swimming, fight etc.[1]

Pathophysiology lies in the fact that extrusive forces impinging on the teeth, when severe enough, can cause a tooth to be displaced out of its socket. For this to happen, the periodontal ligament would have ruptured with remnants remaining on the cementum of the root of the tooth and the inner walls of the alveolar socket. The vessels entering the pulp through the apical foramen would also have been severed with cessation of blood supply to the pulp. The extent of injury sustained by the periodontal ligament and the pulp, and the subsequent healing of these tissues will depend on the extra-alveolar period i.e. the time remains out of its socket and the handling of the tooth.[17]

Treatment is directed at avoiding or minimizing the resultant inflammation from the two main consequences of the avulsed tooth-namely, attachment damage and pulpal infection. The best outcome for a tooth avulsion is when the tooth can be replanted. within a few minutes after the accident. A very high percentage of teeth replanted within 15 minutes will have the PDL restored within a few weeks.[1]

Clinical and radiographic follow-up of avulsed teeth is essential to define prognosis and should be kept for longer periods between 5 and 10 years. In addition to resorption control, other treatment needs arise in the meantime, such as the correction of malocclusions and color changes of traumatized teeth.[18] Orthodontic movements of traumatized teeth have received relatively little attention in the literature, and there is a lack of clinical protocols for their management.[19] Trauma is one of the most common causes of crown darkening,[20] as it generally causes pulp hemorrhage that spreads through the tubules. Hemolysis causes the release of iron sulfide, which is responsible for crown darkening. Abbott and Heah state that internal tooth whitening in devitalized traumatized teeth is generally successful, often after one session only. These authors did not find any cases of external inflammatory resorption in their study.[21]

The present case report describes the management of an avulsed permanent anterior tooth by replantation and functional splinting followed by endodontic treatment.

\section{Materials \& Methods}

A 10 year-old girl was referred to the my private pediatric dental clinic for the treatment of traumatically avulsed maxillary left central incisor after a car accident. The tooth had been found 4 hours after the accident. The upper right central incisor tooth also suffered from luxation. Thereafter, the tooth was replanted by a general practitioner after $30 \mathrm{~min}$. Fig 1a-b

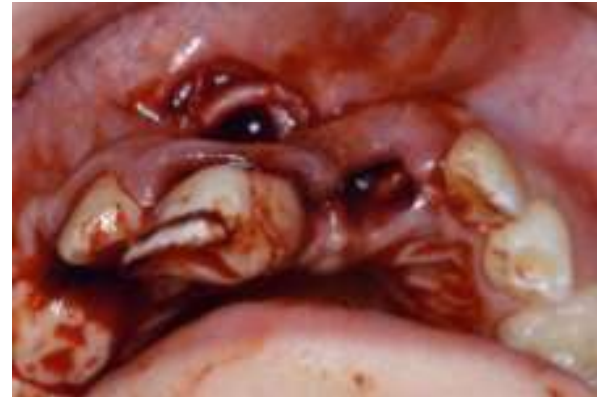

a

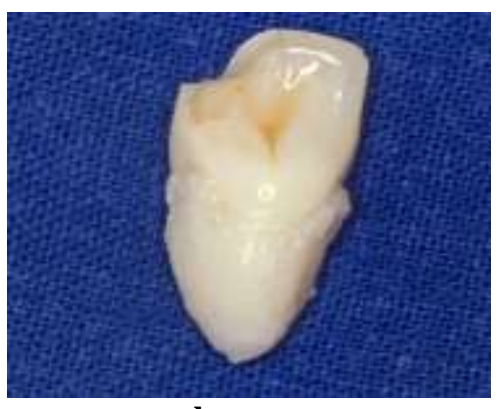

b

Fig 1a-b: Anterior trauma by 10 year old patient. A total luxation of the teeth 11 and 12 with violation of soft tissues

The dentist rinsed the socket and tooth with saline and prophylactic antibiotic therapy had been prescribed for one week. The patient was examined clinically and radiographically. An intraoral examination showed no laceration, abrasions, and contusion of the soft tissues. Maxillary central incisors were wire splinted with acid etch composite resin attached to the adjacent teeth for one week. Fig. 2a-f

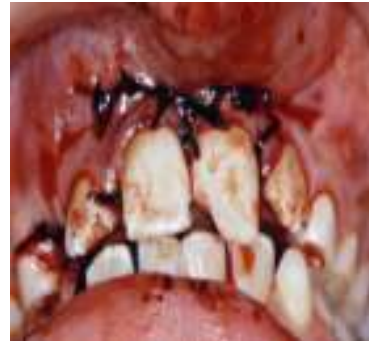

a

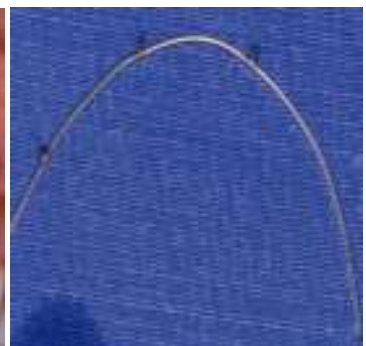

b

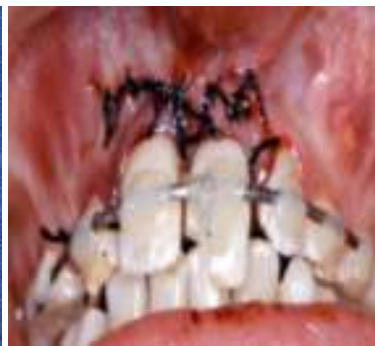

c 

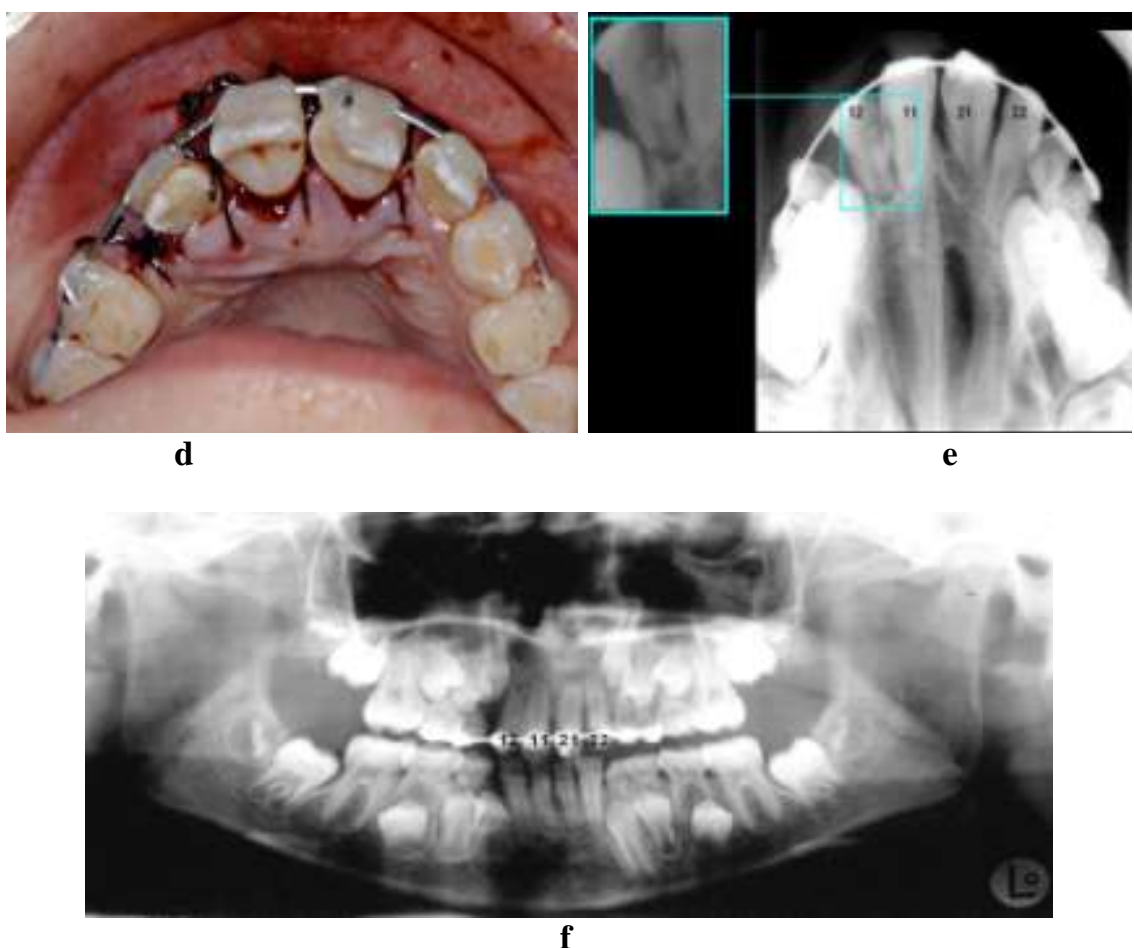

Fig. 2a-f

a-d: Reimplantation of total luxated teeth, for stabilizing a composite wire splint was used e, f: Radiographs after Rimplantion the luxated teeth

The patient returned only 3 months after trauma, on which occasion clinical and radiographic evaluations, as well as splint removal, were performed. A slight mobility and a slight darkening of the crown were found, and radiographic evidence suggested inflammatory resorption at the apical end of tooth 21 . Chemical- mechanical preparation of the root canal was Follow- up was continued every 3 months, and after 1 year of calcium hydroxide intracanal medication use, because of the beneficial properties, no radiographic evidence of external inflammatory resorption areas was found; a final endodontic obturation procedure was then performed. Gray MTA was manipulated according to manufacturer's recommendations and placed in the canal with a lentulo spiral drill.[20] After a year and a half posttrauma, orthopedic treatment was initiated with the use of orthodontic expanders, at the same time, it was made filling with MTA cement. An extension plate of the maxilla and mandible was installed on both dental arches due to a diagnosed atresia, and special care was taken to avoid orthodontic force on replanted tooth 21 . Clinical and radiographic follow- up were continued at 3- month intervals Fig. 3a, b.

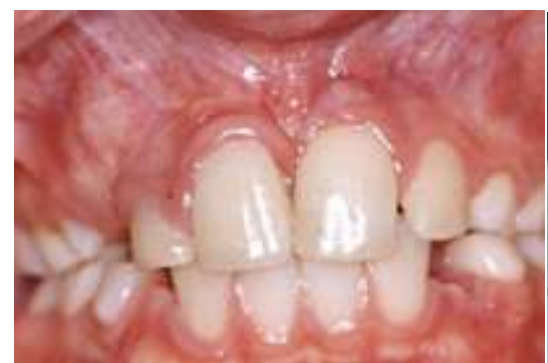

a

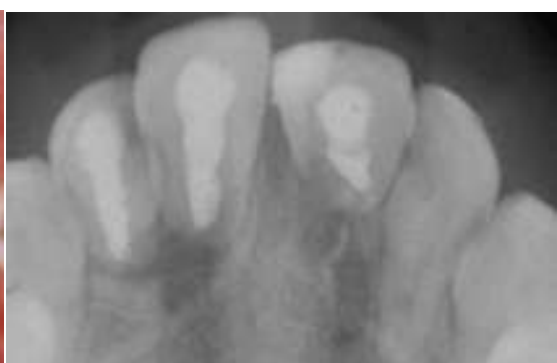

b

Fig. 3a, b:

a: Radiograph after 2.5 years, heavy resorptionb: Clinical situation after 2, 5 years from the accident

Six months after the installation of orthodontic appliances, the patient presented a large enamel crack on tooth 21 due to a new trauma subluxation of the tooth11 that had occurred during sports practice. [20]Radiographically, it was detected the disappearance of the lamina dura at the apical end at the mesial aspect, which was suggestive of replacement resorption. During the clinical and radiographic follow- up visits, the 
stabilization of the ankylosis area was noted. A coronary darkening of the left central incisor was observed clinically. Esthetic compromising, then became the patient's main complaint.

At that point maxillary expansion was completed, and internal dental whitening was performed; at first, an initial color sampling was done; then, following absolute isolation, cervical sealing with light- cured glass ionomer at a $2 \mathrm{~mm}$ distance from the dentin enamel junction was done. Whiteness hydrogen peroxide 35\% whitening agent ) was used in three 15- min applications per session during two clinical consultations. After chromatic evaluation, the efficacy of the whitening procedure was verified, and final sealing of the crown opening with light- cured composite resin was again made possible . Fig. 4a-c

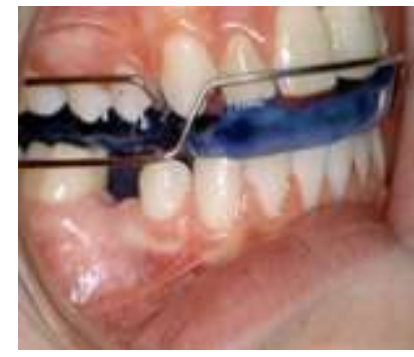

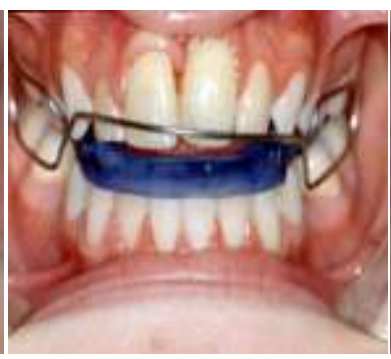

b

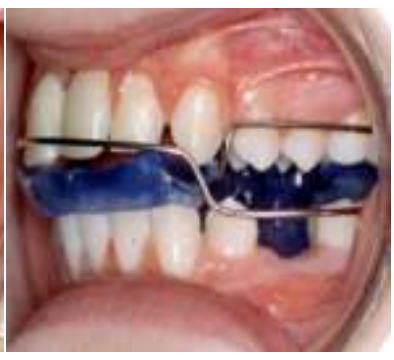

Fig. 4a-c: Intraoral photos during orthodontic treatment-it was only used removable appliances

The case has had a 8 years follow- up to the present time, and the tooth is functional, shows regular mobility, and there is no visible evidence of inflammatory resorption at root leve . The replacement resorption area has stabilized, and a better esthetic result. Fig. 5a-e

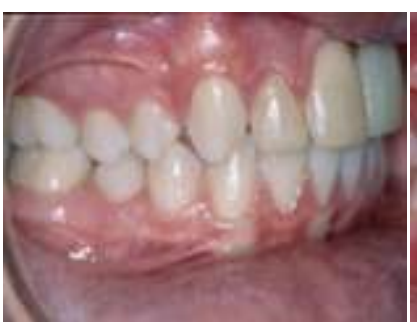

A

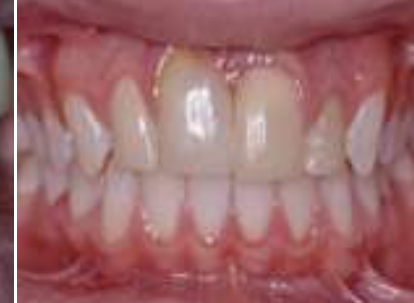

b

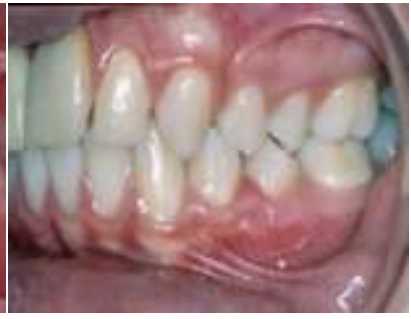

c

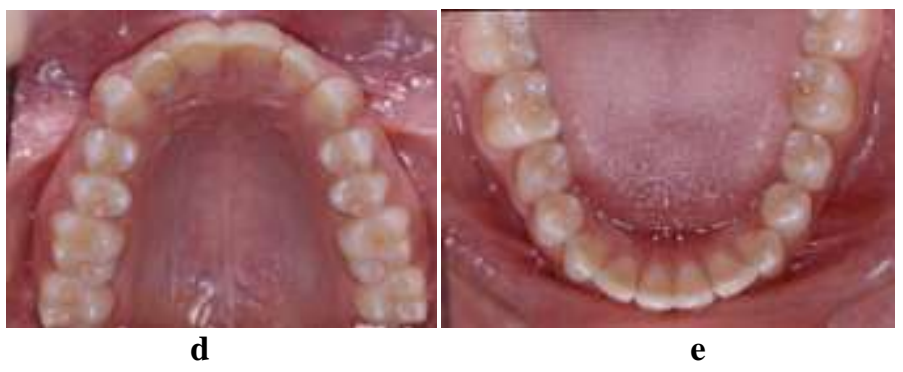

Fig. 5a-e:

\section{Intraoral and extraoral treatment results after the end of the orthodontic treatment}

The patient was kept under follow - up for 8years. No signs of root resorption were seen radiographically during the follow up period when examined clinically,and radiographically imaging.

\section{Discussion}

The success rate of autogenous tooth transplantation in initial reports has been approximately $50 \%$ and therefore, this procedure was not a common treatment option for patients. In addition, etiology of root resorption in transplanted autogenous teeth was not widely understood. In the past few decades, root resorption, its prevention and the healing process of periodontal tissues have been examined by a host of investigators and the success rate of transplantation procedure has increased rapidly from $67 \%$ to $84 \%$. Like other surgical procedures, careful case selection and treatment planning is essential for successful autotransplantation. The donor tooth and recipient site should be examined meticulously for suitability and fit . 
Nothing is known about the long-term success of reimplanted permanent teeth, whether or not the root of the reimplanted tooth will undergo "physiologic" resorption in the new location [20]. But not much data is available about it. Even though long-term treatment results are lacking, reimplantation of permanent central incisor is seen as a promising attempt to reposition a severely malaligned tooth and maintain the surrounding tissues. From transplantations of dilacerated teeth and extreme cases of root fractured avulsed teeth; it is known that about $2 \mathrm{~mm}$ of autologous root length are enough for successful replantation.

In cases of dental avulsion, the traumatology service of the dental school carries out monitoring of the appearance of resorption areas through weekly radiographs. It is worth mentioning that the Service now follows the IADT procedure that recommends the beginning of endodontic therapy between 7 and 10 days after trauma for teeth with a closed apex. External inflammatory resorptionsadvance rapidly in young patients[2] and can lead to serious sequels, what fortunately did not occur in this case.

Trope and Fried man studied histologically the periodontal healing and root resorption of replanted dog teeth stored in viaspan for different time period and compared these healing pattern to those after storage in milk or hanks balance salt solution. The extracted teeth were divided into 4 groups composing 4 teeth each are placed in vials containing viaspan or milk for $6,12,24,36$ hours and viaspan or hanks balanced salt solution for $36,48,72 \& 98$ hours after which they are replanted, and were examined histologically for healing of the supporting tissues. They concluded that for viaspan neither replacement nor inflammatory root resorption was seen after 6 and 12 hours storage. A statistically significant rise in the incidence of replacement resorption was seen at 24 and 36, 48 hours to levels, equal to storage for $6 \& 12$ hours. The occurrence of inflammatory root resorption were low and significantly increased only at 48 hours after teeth it decreased significantly again. Viaspan proved superior to milk as a storage medium. Teeth stored in hanks balance salt solution showed healing, result was similar to those stored in viaspan trope.[22]

Kitzis and Miller, reported replantation of avulsed tooth after prolonged storage. This report demonstrates that if an intact avulsed teeth is retrieved stored carefully and can be treated successfully even after more than 42 hours outside the alveolus.[23]

Gamson, Dumsha, Sydiskis studied the effect of drying time on periodontal ligament cell viability. They studied various dry storage time ranging from 0 to 120 minutes followed by 45 minutes storage in milk. A dual labeling fluorogenic labeling techniques was utilized in under to discriminate this viable and non-viable cell. It was found that at 30 minutes of dry storage there was a statistically significant decrease in the members of viable periodontal ligament cells whencompared to 10 and $20 \mathrm{~min}$ of dry time. They concluded that milk is a suitable storage media for maintaining

periodontal ligament cell viability.[24]

Oikarinen and seppa studied the effect of preservation media on proliferation and collagen biosynthesis of cultured human periodontal ligament. Fibroblast proliferation of cells was studied after 60 minutes incubation in Dulbeccos modified Eagles medium (DMEM), milk, saliva, tap water. They concluded that milk and saliva were superior to water in maintaining these cell functions. [25] Adil N. F reported a case where replantation was done after 36 hours of aulsion in which the root canal was done extraorally. $\mathrm{He}$ explained the importance of vitality of PDL and also the stability of the replanted tooth was better when splinting was done with orthodontic brackets and Australian wire.[26]

Yin- Hua Zhao, Min Zhang found that the PRF induced a significant and continuous stimulation of proliferation in human PDLSCs throughout the 7-day incubation period. The PRF suppressed the osteoblastic differentiation of PDLSCs by decreasing ALP activity and the gene expression of BSP and OCN while upregulating the mRNA expression levels of Col-I and CP23 during the testing period. They suggested that the PDLSCs/PRF construct may be a useful tool for alveolar surgery that has the potential to improve the clinical outcomes in future avulsed tooth reimplantations.[27]

Shweta Jain reported a case of delayed replantation of avulsed teeth after extended extraoral period and nonphysiological storage. This article presented a alternative treatment modality to immediately restore esthetic and function as well as to promote the growth of alveolar crest for proper eruption of adjacent unaffected teeth until a definite prosthetic treatment seems appropriate.[28]

Panzarini et al., in a previous study, demonstrated that the average time range of other reimplanted teeth was between 1 to 4 hours, after which the success of reimplantation was determined by the medium of storage.[11] Success has also been reported in a study where the tooth was reimplanted after 8 hours, but was stored in Euro- Collins solution. Studies have shown that even 10 minutes of dry storage results in desiccation of the periodontal ligament cells and eventual loss of the tooth.[29]

Other studies by Day and Duggal showed that reimplantation after 60 minutes in no storage medium with extraoral endodontics may result in retention of the tooth.[30] However, studies by Petrovic et al. showed that reimplanted dry teeth in dry storage between 15 minutes to 9 hours have low survival rates and a reduced chance of retention. [31]The observation of some clinicians is that if an avulsed tooth is 
not stored in an adequate storage medium, it invariably leads to necrosis of the pulp, periodontal ligament and cementum, and fails to reimplant. [30] The most practical and available storage media are saliva and cold milk which could prevent desiccation of the periodontal ligament cells for up to 60 minutes However, in these case reports, no adequate storage media were used to keep and transport the teeth to the clinic before reimplantation and the results were successful.[29-31]

The literature is indeed very consistent in this regard. Dental trauma does not contraindicate orthodontic treatment, provided there are no pulp or periodontal changes and/or root resorption. However, some studies indicate that traumatized teeth are more predisposed to resorption. But these findings are not conclusive because the final outcome of traumatized tooth treatment can take more than five years to surface. This is one of the factors hampering the analysis of the effects of orthodontic movement on traumatized teeth. These studies involve a small number of patients, who present with different types of injuries and are treated by different researchers using a variety of orthodontic appliances. These factors combine to render inconclusive any findings regarding the effects of orthodontic treatment on traumatized teeth.[32]

According to Malmgren et al,1 most root resorption after reimplantation occurs during the first year posttrauma. [32]Boyd, Kinirons and Gregg found that a time span ranging between 102 and 997 days 3 elapsed before root resorption was detected, suggesting the need for a longer follow-up period before starting orthodontic treatment.[33]

A multidisciplinary tooth trauma approach is indispensable because many patients also have other dental treatment needs, such as in malocclusion cases when orthodontic intervention is needed. Every orthodontic treatment is a resorption risk factor, as an apical inflammation may develop due to the pressure applied to the root during movement, producing ischemic necrosis of the periodontal ligament; [1-5,34,35,36 ] in this case study, the patient had maxillary atresia. Installation of an upper and lower expansion was chosen because of the favorable growth phase it was in; also, it avoided pressure on the traumatized tooth. It must be emphasized that the minimum 1-year period after resorption stagnation recommended by Andreasen and Andreasen [37,38,39 ] so as to begin orthodontic movement was observed. In agreement with the findings by Tondelli et al., 2010, this study also realized the difficulties professionals have in treating traumatized teeth orthodontically, which may be due to the lack of protocols in literature to deal with this specific situation.[ $5,39,40,41]$

\section{Conclusion}

In conclusion, our study suggest that reimplantation of an avulsed tooth as soon as possible and using appropriate splint for such a case, and follow up after treatment by a dentist show good prognosis. The present case report shows that the multidisciplinary approach in the replantation of the avulsed teeth enables the reestablishment of function and esthetics.

\section{References}

[1]. Abu-Hussein M ., Watted N ., and Abdulgani A .; Replantation of Avulsed Permanent Anterior Teeth: A Case Report, RRJDS 2014, Vol 2 ( 4$\}$, 43-52

[2]. Muhamad AH, Azzaldeen A (2012) Autotransplantation of Tooth in Children with Mixed Dentition. Dentistry 2:149. doi:10.4172/2161-1122.1000149

[3]. Abu-Hussein M. , Watted N .,Abdulgani M ., Abdulgani Az .; Tooth Autotransplantation; Clinical Concepts Journal of Dental and Medical Sciences2016, Vol 15 (7) 105-113 DOI: 10.9790/0853-15078105113

[4]. Muhamad AH, Azzaldeen A. Autotransplantation of tooth in mixed dentition- A review. Int. J. Dent.Clinics. 2013;5(1):20-23

[5]. Abu-Hussein M ,1, Nezar W. , Azzaldeen A. , Abdulgani M.; Prevalence of Traumatic Dental Injury in Arab Israeli Community, Journal of Dental and Medical Sciences2016, 15 (7) , 91-98 DOI: 10.9790/0853-150719198

[6]. Lenstrup K, Skieller V. A follow-up study of teeth replanted after accidental loss Acta Odontol Scand 1959;17:503-9

[7]. Andreasen JO, Hiorting-Hansen-E. Replantation of teeth: I. Radiographic and clinical study of 110 human teeth replanted after accidental loss. Acta Odontol Scand 1966;24:263-86.

[8]. Andreasen JO, Hiorting-Hansen E. Replantation of teeth: II. Historical study of 22 replanted anterior teeth in humans. Acta Odontol Scand 1966;24:287-306

[9]. Cvek M, Granath L-E, Hollander L. Treatment of non-vital permanent incisors with calcium hydroxide: III. Variations of occurrence of ankylosis of reimplanted teeth with duration of extra-alveolar period and storage environment. Odont Revy 1974;25:43-6.

[10]. Cvek M, Granath L-E, Hollander L. Treatment of non-vital permanent incisors with calcium hydroxide: III. Variations of occurrence of ankylosis of reimplanted teeth with duration of extra-alveolar period and storage environment. Odont Revy 1974:25:43-6.

[11]. Lindskog S, Peirce AM, Blomlof L, Hammarstrom L. The role of the necrotic periodontal membrane in cementum resorption and ankylosis. Endod Dent Traumatol 1985;1:96-101.

[12]. Andreasen JO. A time-related study of root resorption activity after replantation of mature permanent incisors in monkey. Swed Dent J 1980;4:101-10.

[13]. Andreasen JO. Relationship between cell damage in the periodontal ligament after replantation and subsequent development of root resorption: a time-related study in monkeys. Acta Odontol Scand 1981;39:15-25.

[14]. Matsson L, Andreasen JO, Cvek M, Granath L-E. Ankylosis of experimentally reimplanted teeth related to extra-alveolar period and storage environment. Pediatr Dent 1982;4:327-9. 
[15]. Krasner P, Rankow H, Ehrenreich A. Apparatus for storing and transporting traumatically avulsed teeth. Compend Cont Dent Educ 1989;10:232-7.

[16]. Trope M, Freidman S. Periodontal healing of replanted dog's teeth stored in ViaSpan, milk, Hank's balanced salt solution. Endo Dent Traumatol 1992;8:183-8.

[17]. Andreasen JO, Andreasen F. Textbook and color atlas of traumatic injuries to the teeth. 4th Edition. Copenhagen: Denmark: Munksgaard; 2007. pp.444.

[18]. Tondelli PM, Mendonça MR, Cuoghi OA, Pereira AL, Busato MC. Knowledge on dental trauma and orthodontic tooth movement held by a group of orthodontists. Braz Oral Res 2010;24:76-82

[19]. Goldberg M, Grootveld M, Lynch E. Undesirable and adverse effects of tooth-whitening products: A review. Clin Oral Investig 2010;14:1-10.

[20]. Abu-Hussein M , Abdulgani A , Abdulgani M , Jabareen A.;Single-Step Apexification with Mineral Trioxide Aggregate (MTA) Case Reports, Journal of Dental and Medical Sciences2016. 15(2), 49-53DOI: 10.9790/0853-15284953

[21]. Kinoshita S, Kojima R, Taguchi Y, Noda T. Tooth replantation after traumatic avulsion: A report of ten cases. Dent Traumatol 2002;18:153-6

[22]. Trope M; Clinical management of the avulsed tooth: present strategies and future directions. DentTraumatol 2002; 18: 1-14.

[23]. Kitzis and Miller; Replantation of an avulsed tooth after prolonged storage. Dent Traumatol 1999; 21:15-8.

[24]. Gamson EG, Dumsha TG, Sydiskis R; The effect of drying time of periodontal ligament cell vitality. J Endod $1992 ; 4: 125-136$.

[25]. Oikarinen KS, Seppa ST; Effect of preservation media on proliferation and collagen biosynthesis of periodontal ligament fibroblasts. Endod Dent Traumatol $1987 ; 3: 95-9$.

[26]. Paul Chalakkal, Abi Mathew Thomas, Francis Akkara, Kristlee Sabrin Fernandes. Delayed Replantation after Endodontic and Fluoride Treatment: A 5-Year Follow-up. Int J Clin Pediatric Dent 2011;4:228-231

[27]. in Hua Zhao and Min Zhang; The combined use of cell sheet fragments of periodontal ligament stem cells and platelet-rich fibrin granules for

[28]. avulsed tooth reimplantation. Biomaterials 2013; 34:5506-5520.

[29]. Shweta Jain, Vijay Agarwal, Arun Kumar Gupta, Pramod Prabhakar. Replantation of Immature Avulsed Teeth with Prolonged Extraoral Dry Storage: A Case Report. Int J Clin Pediatric Dent 2012;5:68-71.

[30]. Panzarini SR, Gulineli JL, Poi WR, Sonoda CK, Pedrini D, Brandini DA. Treatment of root surfacein delayed replantation: A review of the literature. Dent Traumatol 2008;24:277- 82.

[31]. Day P, Duggal M. Interventions for treating traumatized permanent front teet

[32]. Avulsed (knocked out) and replanted. Cochrane Database Syst Rev 2010;20:

[33]. CD006542.

[34]. Petrovic B, Markovic D, Peric T, Blagojevic D. Factors related to treatment and

[35]. outcomes of avulsed teeth. Dent Traumatol 2010;26:52- 9.

[36]. Malmgren B, Cvek M, Lundberg M, Frykholm A. Surgical treatment of ankylosed and infrapositioned reimplanted incisors in adolescents. Scand J Dent Res. 1984 Oct;92(5):391-9.

[37]. Kinirons MJ, Boyd DH, Gregg TA. Inflammatory and replacement resorption in reimplanted permanent incisor teeth: a study of the characteristics of 84 teeth. Endod Dent Traumatol. 1999 Dec;15(6):269-72.

[38]. Kindelan SA, Day PF, Kindelan JD, Spencer JR, Duggal MS. Dental trauma: An overview of its influence on the management of orthodontic treatment. Part 1. J Orthod 2008;35:68-78.

[39]. Abu-Hussein M, , Abdulgani Azzaldeen; Intentional replantation of maxillary second molar; case report and 15-year follow-up. Journal of Dental and Medical Sciences. 2016, Vol 15, 1, PP 67-73 DOI: 10.9790/0853-15126773

[40]. Abu-Hussein M., Abdulgani A., Watted N .Zahalka M.; Congenitally Missing Lateral Incisor with Orthodontics, Bone Grafting and Single-Tooth Implant: A Case Report. Journal of Dental and Medical Sciences2015, 14(4),124-130 DOI: 10.9790/08531446124130

[41]. Abu-Hussein M ,1, Nezar W. , Azzaldeen A. , Abdulgani M.; Prevalence of Traumatic Dental Injury in Arab Israeli Community, Journal of Dental and Medical Sciences2016, 15 (7) , 91-98 DOI: 10.9790/0853-150719198

[42]. Abdulgani A.,. Kontoes N., Chlorokostas G.,Abu-Hussein M .;Interdisciplinary Management Of Maxillary Lateral Incisors Agenesis With Mini Implant Prostheses: A Case Report; IOSR-JDMS 2015,14 (12), 36-42 DOI: 10.9790/0853-141283642

[43]. Abusalih A. , Ismail H , Abdulgani A. , Chlorokostas G ., Abu-Hussein M .; Interdisciplinary Management of Congenitally Agenesis Maxillary Lateral Incisors: Orthodontic/Prosthodontic Perspectives, Journal of Dental and Medical Sciences2016, 15 ( 1) 90-99 DOI: 10.9790/0853-15189099

[44]. Abu-Hussein M., Watted N., Abdulgani A., BorbélyB.; Modern Treatment for Congenitally Missing Teeth : A MultidisciplinaryApproach; INTERNATIONAL JOURNAL OF MAXILLOFACIAL RESEARCH,2015,1(2);179-190

[45]. Abu-Hussein M, Chlorokostas G, Watted N , Abdulgani A , Jabareen A.,Pre-Prosthetic Orthodontic Implant for Management of Congenitally Unerupted Lateral Incisors - A Case Report Journal of Dental and Medical Sciences 2016 .Vol 15 (2 ), 99-104 DOI: $10.9790 / 0853-152899104$ 